\title{
Convex Dimension of Locally Planar Convex Geometries
}

\author{
W. D. Morris, Jr. \\ Department of Mathematical Sciences, George Mason University, \\ Fairfax, VA 22030, USA \\ wmorris@gmu.edu
}

\begin{abstract}
We prove that convex geometries of convex dimension $n$ that satisfy two properties satisfied by nondegenerate sets of points in the plane, may have no more than $2^{n-1}$ points. We give examples of such convex geometries that have $\left(\begin{array}{l}n \\ 4\end{array}\right)+\left(\begin{array}{l}n \\ 2\end{array}\right)+\left(\begin{array}{l}n \\ 0\end{array}\right)$ points.
\end{abstract}

\section{Introduction and Terminology}

The problems we consider can be stated in terms of elementary convexity properties of finite sets of points in general position in the plane. These properties are described in terms of the convex geometry (also called antimatroid) realized by the point set.

Definition 1.1. A convex geometry is a pair $(X, \mathcal{C})$, where $X$ is a finite set and $\mathcal{C}$ is a collection of subsets of $X$ satisfying:

1. $X \in \mathcal{C}$.

2. For every $A \in \mathcal{C}, A \neq X$ there is an $x \in X \backslash A$ so that $A \cup\{x\} \in \mathcal{C}$.

3. $A, B \in \mathcal{C}$ implies $A \cap B \in \mathcal{C}$.

A good reference for convex geometries is [2]. A convex geometry $(X, \mathcal{C})$ is realizable in $\mathbb{R}^{d}$ if there is a function $\varphi: X \rightarrow \mathbb{R}^{d}$ so that for any $A \subseteq X$ we have $A \in \mathcal{C}$ if and only if $\varphi(A)=K \cap \varphi(X)$ for some convex set $K$ in $\mathbb{R}^{d}$. In this case we say that $\varphi(X)$ realizes $(X, \mathcal{C})$.

Let $E$ be a finite set and let $\mathcal{L}=\left\{L_{e}: e \in E\right\}$ be a collection of linear orders of $X$. For $x, y \in X, x \neq y$, define $S_{y x}=\left\{e \in E: y<x\right.$ in $\left.L_{e}\right\}$. Define a collection $\mathcal{C}$ as follows: $A \in \mathcal{C}$ if and only if there is no $x \in X \backslash A$ so that $\bigcap_{y \in A} S_{y x}=\emptyset$. The pair $(X, \mathcal{C})$ constructed this way is a convex geometry [2, Theorem 5.1]. We say that the set $\mathcal{L}$ generates $\mathcal{C}$. Every convex geometry can be constructed this way [2, Theorem 5.2]. The smallest number of linear orders needed to generate a convex geometry $(X, \mathcal{C})$ in this manner is called [4] the convex dimension of $(X, \mathcal{C})$. If $X$ is a set of points in the plane 
that realizes $(X, \mathcal{C})$, we refer to the convex dimension of $(X, \mathcal{C})$ simply as the convex dimension of $X$.

Example 1.2. Suppose $X=\{x, y, z, w\}, x, y$, and $z$ are vertices of a triangle, and $w$ is in the interior of this triangle. In the convex geometry $(X, \mathcal{C})$ realized by $X, \mathcal{C}$ is the collection of all subsets of $X$ except $\{x, y, z\}$. Let $L_{1}$ be the order $z<y<w<x$, let $L_{2}$ be the order $x<z<w<y$, and let $L_{3}$ be $y<x<w<z$. The set $\left\{L_{1}, L_{2}, L_{3}\right\}$ generates $(X, \mathcal{C})$. Because $\{y, z, w\} \in \mathcal{C}$, any set of linear orders generating $\mathcal{C}$ must contain an order in which $x$ is largest. For similar reasons, any such set of orders must also contain an order on which $y$ is largest and one in which $z$ is largest. Thus the convex dimension of $X$ is 3 .

Problem 1.3. Determine the largest number of points that a set $X$ in the plane may have if no three points of $X$ are on a line and the convex dimension of $X$ is $n$.

Denote by $M_{\text {real }}(n)$ the largest cardinality of a set $X$ of points in general position in the plane with convex dimension $n$. We show in Section 3 that $M_{\text {real }}(n) \leq 2^{n-1}$.

Problem 1.3 is a close relative of a famous problem of Erdős and Szekeres [6]:

Problem 1.4. Determine the largest number of points that a set $X$ in the plane may have if no three points of $X$ are on a line and $X$ does not contain the vertex set of a convex $(n+1)$-gon.

Erdôs and Szekeres [7] found examples of $2^{n-1}$ points, in general position in the plane, that contain no $(n+1)$-gon. It is widely believed that no larger sets exist, but the best known upper bound [18] is $|X| \leq\left(\begin{array}{c}2 n-3 \\ n-2\end{array}\right)+1$. The paper [15] studies the problem of Erdős and Szekeres in the context of convex geometries.

A convex geometry $(X, \mathcal{C})$ with $|X|>d$ is $d$-free if every $d$-element subset of $X$ is in $\mathcal{C}$. Note that a convex geometry with at least three points that is realizable in $\mathbb{R}^{2}$ is 2 -free if and only if no three points of a realization are on a line.

The members of a convex geometry $\mathcal{C}$ are called closed sets. Every convex geometry $(X, \mathcal{C})$ defines a closure operator $c l_{\mathcal{C}}$. If $A$ is a subset of $X, c l_{\mathcal{C}}(A)$ is the smallest member of $\mathcal{C}$ that contains $A$.

Definition 1.5. A convex geometry $(X, \mathcal{C})$ is called locally planar if for each $x \in X$ there is a function $\varphi_{x}: X \rightarrow \mathbb{R}^{2}$ that satisfies, for each $A \subseteq X, x \in c l_{\mathcal{C}}(A)$ if and only if $\varphi_{x}(x)$ is in the convex hull of $\left\{\varphi_{x}(y): y \in A\right\}$.

Note that if $(X, \mathcal{C})$ is realized by a set $\varphi(X)$ in the plane, then we may take $\varphi_{x}=\varphi$ for each $x \in X$ to show that $(X, \mathcal{C})$ is locally planar. We will see that not every locally planar convex geometry is realizable in the plane.

Denote by $M_{\mathrm{lp}}(n)$ the largest number of points that a 2 -free locally planar convex geometry of convex dimension $n$ may have. We show in Section 2 that $M_{\mathrm{lp}}(n) \leq 2^{n-1}$, and hence that $M_{\text {real }}(n) \leq 2^{n-1}$.

A subset $A$ of $X$ is called independent if $a \notin c l_{\mathcal{C}}(A \backslash a)$ for all $a \in A$. If $(X, \mathcal{C})$ is generated by a set $\mathcal{L}$ of linear orders of a set $X$, and $A \subseteq X$, then independence of $A$ is 
equivalent to the requirement that each element of $A$ be greater than the other elements of $A$ in at least one of the orders of $\mathcal{L}$. It follows that the convex dimension is at least as large as the size of a largest independent subset of $X$. If $(X, \mathcal{C})$ is realized by a set $\varphi(X)$ in the plane, then a subset $A \subseteq X$ is independent if and only if $\varphi(A)$ is the set of vertices of a convex polygon.

The appearance of the function $2^{n-1}$ in the bounds for both Problems 1.3 and 1.4 suggests a closer relationship between these problems. Closer inspection in Section 3, however, reveals that $M_{\mathrm{lp}}(n)<2^{n-1}$ when $n>5$. We conjecture that $M_{\mathrm{lp}}(n)=\left(\begin{array}{l}n \\ 4\end{array}\right)+$ $\left(\begin{array}{l}n \\ 2\end{array}\right)+\left(\begin{array}{l}n \\ 0\end{array}\right)$. Furthermore, there is a unique 2-free locally planar convex geometry with convex dimension 5 and 16 points, and this convex geometry is not realizable. Thus $M_{\text {real }}(5)<M_{\mathrm{lp}}(5)$, and it seems reasonable to conjecture that $M_{\text {real }}(n)$ is at most a polynomial of degree 3 .

The convex geometry of convex dimension 5 and 16 points constructed in Section 3 is a member of a family of locally planar convex geometries of convex dimension $n$ that are not realizable in the plane, but are realizable by uniform rank 3 oriented matroids. We give the necessary background for oriented matroids in Section 4.

Section 5 shows how to construct uniform oriented matroids of rank 3 that realize locally planar convex geometries that have convex dimension $n$ and $\left(\begin{array}{l}n \\ 4\end{array}\right)+\left(\begin{array}{l}n \\ 2\end{array}\right)+\left(\begin{array}{l}n \\ 0\end{array}\right)$ points. The elements of these convex geometries are regions of a disk that is cut up by line segments connecting a set of $n$ points on the boundary of the disk. These examples are interesting from the viewpoint of oriented matroid theory. The $n=5$ instance meets the conjectured upper bound for Goodman and Pollack's "pseudoline" generalization [9] of Problem 1.4. This example has some appealing symmetries that the corresponding example of [7] does not have (at the price of nonrealizability.)

\section{An Upper Bound}

Throughout this section we assume that $(X, \mathcal{C})$ is a 2-free convex geometry on $X$. We also assume that $\mathcal{L}=\left\{L_{e}: e \in E\right\}$ is a finite set of linear orders of $X$ that generates $(X, \mathcal{C})$. For each $x \in X$, define $D_{x}=\left\{S_{y x}: y \neq x\right\}$.

Example 2.1. In Example 1.2 of Section 1, we have $D_{x}=\{\{1\},\{1,3\}\}, D_{y}=$ $\{\{2\},\{1,2\}\}, D_{z}=\{\{3\},\{2,3\}\}$, and $D_{w}=\{\{1,2\},\{1,3\},\{2,3\}\}$. Note that all sets in $D_{x}$ contain $\{1\}$, all sets in $D_{y}$ contain $\{2\}$, and all sets in $D_{z}$ contain $\{3\}$. Each of the sets of $D_{w}$ contains two of the three elements $x, y, w$.

A family of subsets of $E$ is called intersecting if $F \cap G \neq \emptyset$ whenever $F, G \in E$. An intersecting family of subsets of $E$ is maximal if it is contained in no other intersecting family.

Lemma 2.2. For each $x \in X, D_{x}$ is an intersecting family.

Proof. If $S_{y x} \cap S_{z x}=\emptyset$, then $\{y, z\} \notin \mathcal{C}$, contradicting 2-freeness. 
Corollary 2.3. No two families $D_{x}$, for $x \in X$, are contained in the same maximal intersecting family of subsets of $E$.

The corollary follows because, for $x \neq y, S_{y x}$ is the complement in $E$ of $S_{x y}$. The preceding lemma and its corollary are essentially to be found in [12]. We would like to thank Jim Lawrence for pointing out to us that the results of [12] apply to antimatroids. The number $\gamma(n)$ of maximal intersecting families of subsets of an $n$-element set grows very quickly, see [14] and [5]. In particular, $\gamma(4)=12, \gamma(5)=81$, and $\gamma(n)$ is at least $2^{\left(\begin{array}{c}n-1 \\ \lfloor n-1) / 2\rfloor\end{array}\right)}$. The construction in [12] gives a 2-free convex geometry of convex dimension $n$ with $\gamma(n)$ elements, for any $n$.

The convex geometries constructed in [12] are 2-free, but they are very far from being realizable in the plane. We show this by adding to the requirement of 2 -freeness the additional requirement of local planarity. Suppose that $(X, \mathcal{C})$ is 2-free and locally planar. Recall that this means that for every $x \in X$ we have a function $\varphi_{x}: X \rightarrow \mathbb{R}^{2}$ so that for any $A \subseteq X, x \in c l_{\mathcal{C}}(A)$ if and only if $\varphi(x)$ is in the convex hull of $\varphi(A)$. We may clearly assume that $\varphi(x)=0$, the origin, and that $\left|\varphi_{x}(y)\right|=1$ for each $y \in X \backslash x$. Because $(X, \mathcal{C})$ is 2-free, it follows that $\varphi_{x}(y) \neq-\varphi_{x}(z)$ for $y, z \in X \backslash\{x\}$. For $y, z \in X \backslash x$, define $I_{x}(y, z)$ to be the shorter of the two closed $\operatorname{arcs}$ in $S^{1}$ from $\varphi_{x}(y)$ to $\varphi_{x}(z)$. We define a relation $y \sim_{x}$ $z$ if there is no element of $-\varphi(X \backslash x)$ in $I_{x}(y, z)$. The following lemma is easy to verify:

Lemma 2.4. The relation $\sim_{x}$ is an equivalence relation on $X \backslash x$, and the number of equivalence classes is odd.

If there are $2 r+1$ equivalence classes $A_{1}, A_{2}, \ldots, A_{2 r+1}$ for $\sim_{x}$, we can assume that we encounter the sequence $\left(\varphi_{x}\left(A_{1}\right), \varphi_{x}\left(A_{2}\right), \ldots, \varphi_{x}\left(A_{2 r+1}\right)\right)$ as we go clockwise around the unit circle. A subset of $\left\{A_{1}, A_{2}, \ldots, A_{2 r+1}\right\}$ is called consecutive if its image under $\varphi_{x}$ is consecutive in the clockwise ordering.

It is possible, using the results of [3], to define local planarity in terms of combinatorial properties of a function $\varphi_{x}$ from $X \backslash x$ to $S^{1}$, without explicitly referring to the points $-\varphi_{x}(z)$.

Proposition 2.5. Let $w, z \in X$ be such that for every $y \in X \backslash x$, either $\varphi_{x}(y)$ or $-\varphi_{x}(y)$ is in $I_{x}(w, z)$. Then there exists $e \in E$ so that $e \in S_{y x}$ if and only if $\varphi_{x}(y)$ is in $I_{x}(w, z)$.

Proof. (See Fig. 2.1.) Let $A=\left\{y \in X \backslash x: \varphi_{x}(y) \in I_{x}(w, z)\right\}$. Then local planarity implies that $x \in \operatorname{cl}_{\mathcal{C}}(B)$ for any proper superset $B$ of $A$, but $x \notin c l_{\mathcal{C}}(A)$. The definition of a convex geometry now implies that $A$ is in $\mathcal{C}$. Because $A \in \mathcal{C}, \bigcap_{y \in A} S_{y x}$ must be nonempty. Let $e$ be an element of $\bigcap_{y \in A} S_{y x}$. In particular, this means that $e \in S_{z x} \cap S_{w x}$. On the other hand, if $y \notin(A \cup\{x\})$, then local planarity implies that $S_{y x} \cap S_{w x} \cap S_{z x}$ is empty, so $e \notin S_{y x}$.

In the language of convex geometries, the set $A$ of the preceding proof is called a copoint attached to $x$. The number of copoints attached to a point $x$ is called, in [13], the valence of $x$. It is also pointed out in [13] that the number of copoints attached to a point in a 2-free planar convex geometry is odd. 


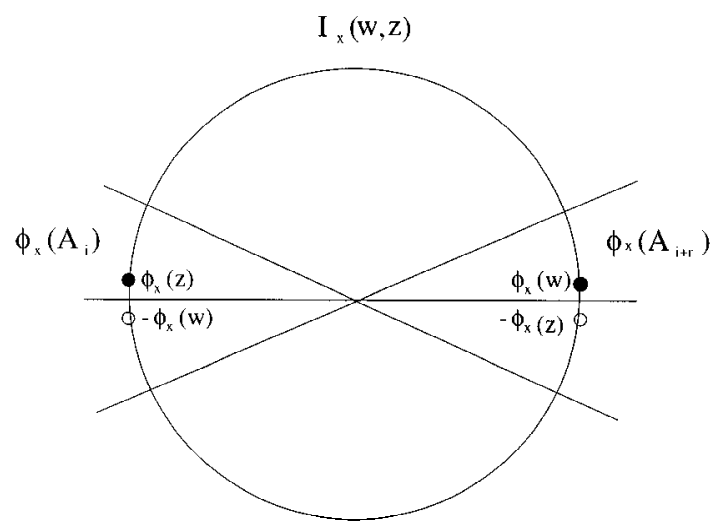

Fig. 2.1. See Proposition 2.5.

Proposition 2.6. Suppose that $\sim_{x}$ has $2 r+1$ equivalence classes for some integer $r$. Then there exists a set $G$ of $2 r+1$ elements of $E$ so that for every $y \in X \backslash x$ we have $\left|G \cap S_{y x}\right|=r+1$.

Proof. For each consecutive set $H$ of $r+1$ equivalence classes we can find $z, w \in X \backslash x$ so that $\varphi_{x}(y) \in I_{x}(w, z)$ if and only if $y$ is in one of the equivalence classes in $H$. We can therefore find an element $e_{H} \in E$ so that $e_{H} \in S_{y x}$ if and only if $y$ is in one of the intervals in $H$. Each $y \in X \backslash x$ is contained in an equivalence class, and each equivalence class is contained in $r+1$ such $H$, so the result follows.

Corollary 2.7. For each $x \in X$ there is an odd subset $\chi(x)$ of $E$ so that $D_{x}$ is contained in the maximal intersecting family of subsets of $E$ obtained by taking all subsets that contain more than half of the elements of $\chi(x)$.

Corollary 2.3 shows that if $x \neq y$, then $\chi(x) \neq \chi(y)$. Because there are $2^{n-1}$ subsets of odd size of a set of $n$ elements, we have the following:

Theorem 2.8. Suppose that $\mathcal{C}$ is a 2-regular locally planar convex geometry on a set $X$, and suppose that the convex dimension of $\mathcal{C}$ is $n$. Then $|X| \leq 2^{n-1}$.

\section{Uniqueness Results}

This section is devoted to determining, for small $n$, the locally planar 2-free convex geometries with convex dimension $n$ and $2^{n-1}$ points. Suppose that $(X, \mathcal{C})$ is 2 -free and locally planar, and that there exist linear orders $L_{1}, L_{2}, \ldots, L_{n}$ of $X$ that generate $X$. Section 2 showed that there is a 1-1 function $\chi$ from $X$ to the collection of odd subsets of the set $\{1,2, \ldots, n\}$, so that for every $x \neq y$ in $X$ we have $\left|\chi(x) \cap S_{y x}\right|=(|\chi(x)|+1) / 2$. We assume that $X$ is the collection of all odd subsets of $\{1,2, \ldots, n\}$, and that $\chi$ is the identity function. 
We start with $n=3$. For $i=1,2,3$, the point $\{i\}$ must be largest in order $L_{i}$. This is because $\left|\{i\} \cap S_{y x}\right|$ must be 1 when $x=\{i\}$ and $y \neq\{i\}$. The point labeled $\{1,2,3\}$ must be larger than $\{i\}$ on exactly two orders, which must be the orders other than $L_{i}$, for $i=1,2,3$. We have thus determined the orders $L_{1}, L_{2}, L_{3}$ up to the order of the least two elements of each order. The order of these least two elements is, however, irrelevant to determining $\mathcal{C}$. We see that $\{1,2,3\} \in \operatorname{cl}_{\mathcal{C}}(\{\{1\},\{2\},\{3\}\})$ and hence $\{\{1\},\{2\},\{3\}\} \notin \mathcal{C}$, but all other subsets of $X$ are in $\mathcal{C}$. Thus $(X, \mathcal{C})$ is realizable by four points in the plane, for which one of the points (labeled by $\{1,2,3\}$ ) is in the convex hull of the other three (as in Example 1.2.)

For $n=4$, we again note that $\{i\}$ must be largest in order $L_{i}$, for $i=1,2,3,4$. The point $\{1,2,3\}$ must be below the point $\{4\}$ on exactly one of $L_{1}, L_{2}$, or $L_{3}$. Without loss of generality, we may assume that $\{1,2,3\}<\{4\}$ in $L_{3}$. It is, however, necessary that both of $\{1,3,4\}$ and $\{2,3,4\}$ are above $\{4\}$ on order $L_{3}$, because they are both below $\{4\}$ on order $L_{4}$. Because $\{1,2,4\}$ must be larger than $\{1,2,3\}$ on exactly one of $L_{1}$ and $L_{2}$, it must be true that $\{1,2,4\}$ is below $\{1,2,3\}$ in $L_{3}$. Generalizing our initial choice of $\{1,2,3\}$ to arbitrary $\{i, j, k\}$, we see that each $\{i, j, k\}$ must be in fifth position in exactly one of the orders $L_{i}, L_{j}, L_{k}$, and in this order, $\{i, j, k\}$ is directly below the point $\{l\}$, where $l \notin\{i, j, k\}$. We therefore have a permutation $\pi:\{1,2,3,4\} \rightarrow\{1,2,3,4\}$, with $\pi(i)=j$ if $\{i\}$ is in the fourth position of $L_{j}$. This permutation has no fixed points, so it is either the product of two disjoint transpositions or it is a cycle of length four. This gives us the two different possibilities for $n=4$. The two possibilities are illustrated below, with realizations of the resulting convex geometries.

Example 3.1. Type I (cycle of length four):

$\begin{array}{cccc}\{1,2,4\} & \{1,2,3\} & \{2,3,4\} & \{1,3,4\} \\ \{1,2,3\} & \{2,3,4\} & \{1,3,4\} & \{1,2,4\} \\ \{2\} & \{3\} & \{4\} & \{1\} \\ \{1,3,4\} & \{1,2,4\} & \{1,2,3\} & \{2,3,4\} \\ \{2,3,4\} & \{1,3,4\} & \{1,2,4\} & \{1,2,3\} \\ \{3\} & \{4\} & \{1\} & \{2\} \\ \{4\} & \{1\} & \{2\} & \{3\}\end{array}$

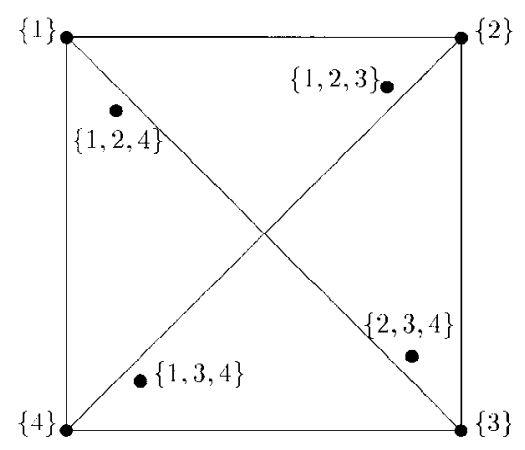


Example 3.2. Type II (two disjoint transpositions):

$\{1\}$ $\{1,2,3\}$

$\{1,2,4\}$

$\{2\}$

$\{1,3,4\}$

$\{2,3,4\}$

$\{3\}$

$\{4\}$
$\{2\}$

$\{1,2,4\}$

$\{1,2,3\}$

$\{1\}$

$\{2,3,4\}$

$\{1,3,4\}$

$\{4\}$

\{3\}
\{3\}

$\{1,3,4\}$

$\{2,3,4\}$

$\{4\}$

$\{1,2,3\}$

$\{1,2,4\}$

$\{1\}$

$\{2\}$
$\{4\}$

$\{2,3,4\}$

$\{1,3,4\}$

\{3\}

$\{1,2,4\}$

$\{1,2,3\}$

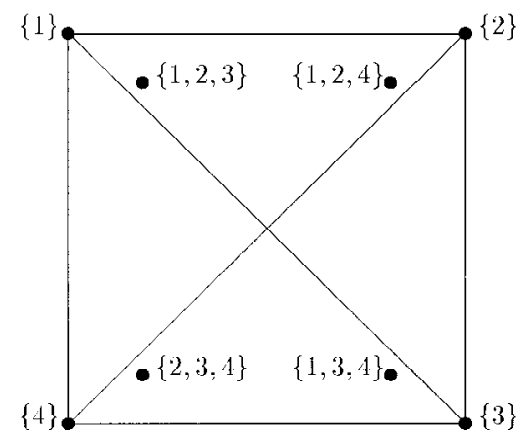

In each case, we may reorder arbitrarily the last three points in any order without changing the convex geometry generated.

The $n=4$ instance of a convex geometry realizable by a nondegenerate set of $2^{n-1}$ points in the plane, with no independent set of size greater than $n$, given in [7] and [16], is of type II.

Next, suppose that we have a 2-regular, locally planar convex geometry $\mathcal{C}$ with 16 points and convex dimension 5 . For each $i=1,2,3,4,5$, we define the convex geometry $\mathcal{C} \backslash i$ to be the convex geometry generated by the orders $L_{j}$ for $j \neq$ $i$, with all elements labeled by subsets containing $i$ deleted. It is easy to see that $\mathcal{C} \backslash i$ must be locally planar for each $i$. Because each $\mathcal{C} \backslash i$ has eight points and convex dimension 4 , it must be of type I or type II. We now show that it cannot be of type II.

Suppose that $\mathcal{C} \backslash 5$ is of type II, and assume that the sets in the orders $L_{1}, L_{2}, L_{3}, L_{4}$ that do not contain 5 are ordered as in Example 3.2. Consider the placement of the sets $\{1,2,5\}$ and $\{3,4,5\}$ in $L_{5}$. Because $\{1,2,5\}$ must be above $\{2\}$ in $L_{1}$ and above $\{1\}$ in $L_{2}$, we must have $\{1,2,5\}$ above $\{3\}$ in both $L_{1}$ and $L_{2}$. This means that $\{1,2,5\}$ is below $\{3\}$ in $L_{5}$. For a similar reason, $\{3,4,5\}$ is below $\{1\}$ in $L_{5}$. However, $\{1,2,5\}>\{1\}$ in $L_{5}$, and $\{3,4,5\}>\{3\}$ in $L_{5}$. This yields the contradiction $\{1,2,5\}>\{1\}>\{3,4,5\}>$ $\{3\}>\{1,2,5\}$ in $L_{5}$.

We can therefore assume that $\mathcal{C} \backslash 5$ is as in Example 3.1, and that each of the convex geometries $\mathcal{C} \backslash i$ for $i=1,2,3,4$ is of type I. Because of the cyclic nature of Example 3.1, we can choose without loss of generality that $\{1\}$ is the largest in $L_{5}$ among the points 
$\{1\},\{2\},\{3\},\{4\}$. Once this choice is made, the type I nature of the $\mathcal{C} \backslash i$ forces the orderings of the following example:

\section{Example 3.3.}

$\begin{array}{ccccc}\{1\} & \{2\} & \{3\} & \{4\} & \{5\} \\ \{1,2,5\} & \{1,2,3\} & \{2,3,4\} & \{3,4,5\} & \{1,4,5\} \\ \{1,2,4\} & \{2,3,5\} & \{1,3,4\} & \{2,4,5\} & \{1,3,5\} \\ \{1,2,3\} & \{2,3,4\} & \{3,4,5\} & \{1,4,5\} & \{1,2,5\} \\ \{2\} & \{3\} & \{4\} & \{5\} & \{1\} \\ \{1,3,5\} & \{1,2,4\} & \{2,3,5\} & \{1,3,4\} & \{2,4,5\} \\ \{1,2,3,4,5\} & \{1,2,3,4,5\} & \{1,2,3,4,5\} & \{1,2,3,4,5\} & \{1,2,3,4,5\} \\ \{2,3,5\} & \{1,3,4\} & \{2,4,5\} & \{1,3,5\} & \{1,2,4\} \\ \{1,3,4\} & \{2,4,5\} & \{1,3,5\} & \{1,2,4\} & \{2,3,5\} \\ \{2,3,4\} & \{3,4,5\} & \{1,4,5\} & \{1,2,5\} & \{1,2,3\} \\ \{3\} & \{4\} & \{5\} & \{1\} & \{2\} \\ \{1,4,5\} & \{1,2,5\} & \{1,2,3\} & \{2,3,4\} & \{3,4,5\} \\ \{2,4,5\} & \{1,3,5\} & \{1,2,4\} & \{2,3,5\} & \{1,3,4\} \\ \{3,4,5\} & \{1,4,5\} & \{1,2,5\} & \{1,2,3\} & \{2,3,4\} \\ \{4\} & \{5\} & \{1\} & \{2\} & \{3\} \\ \{5\} & \{1\} & \{2\} & \{3\} & \{4\}\end{array}$

We may interchange the 10th and 11th points in any order, and we may also reorder the last four points in the orders, without changing the convex geometry generated by these orders.

We now assume that $L_{1}, L_{2}, \ldots, L_{6}$ generate a locally planar convex geometry $\mathcal{C}$ on a set of 32 elements. It follows that each of the convex geometries $\mathcal{C} \backslash i$, for $i=1,2, \ldots, 6$, is isomorphic to the convex geometry of Example 3.3. In particular, we can assume that the subsets of $\{1,2, \ldots, 6\}$ that do not contain 6 are ordered in $L_{1}, L_{2}, \ldots, L_{5}$ as in that example. As in the argument for $n=5$, we can arbitrarily assume that $\{1\}$ is largest in $L_{6}$ of the elements $\{1\},\{2\},\{3\},\{4\},\{5\}$. Consideration of the convex geometries $\mathcal{C} \backslash i$ leads us to conclude that $\{1,3,5\}>\{i+2\}>\{2,4,6\}$ on $L_{i}$ if $i$ is odd, and that $\{2,4,6\}>\{i+2\}>\{1,3,5\}$ on $L_{i}$ if $i$ is even. This, however, contradicts the fact that $\{2,4,6\}$ must be above $\{1,3,5\}$ on $L_{i}$ for some odd $i$. This gives us the following proposition.

Proposition 3.4. There is no 2-regular, locally planar convex geometry of convex dimension $n$ with $2^{n-1}$ elements if $n \geq 6$.

\section{Locally Planar Convex Geometries and Rank 3 Oriented Matroids}

We show in this section that many, and perhaps all, locally planar convex geometries can be realized by acyclic uniform oriented matroids. The standard reference for oriented matroids is [1]. We are only interested in uniform (sometimes called simple) oriented matroids. 
Definition 4.1. A signed set $D$ on a finite set $X$ is an ordered pair $\left(D^{+}, D^{-}\right)$of subsets of $X$, where $D^{+} \cap D^{-}=\emptyset$. We write $\bar{D}=D^{+} \cup D^{-}$and $-D=\left(D^{-}, D^{+}\right)$.

Definition 4.2. A uniform oriented matroid of rank $r$ is a pair $\mathcal{O}=(X, \mathcal{D})$, where $\mathcal{D}$ is a collection of signed subsets of $X$, called the circuits of $\mathcal{O}$, satisfying the following properties:

1. $|X| \geq r$, and if $|X|>r$, then $\mathcal{D} \neq \emptyset$.

2. If $D \in \mathcal{D}$, then $-D \in \mathcal{D}$.

3. If $D_{1}, D_{2} \in \mathcal{D}$ and $\bar{D}_{2} \subseteq \bar{D}_{1}$, then $D_{1}=D_{2}$ or $D_{1}=-D_{2}$.

4. If $D \in \mathcal{D}$, then $|\bar{D}|=r+1$.

5. If $D_{1} \in \mathcal{D}, x \in X$, and $x \notin \bar{D}_{1}$, then there is $D_{2} \in \mathcal{D}$ such that $x \in D_{2}^{+}, D_{2}^{+} \subseteq$ $D_{1}^{+} \cup\{x\}$, and $D_{2}^{-} \subseteq D_{1}^{-}$.

Oriented matroids that satisfy (1)-(5) are called uniform because the sets $\bar{D}$ all have the same cardinality. The axiom system (1)-(5) appears in [8] under the name positivity system.

A uniform oriented matroid $(X, \mathcal{D})$ of rank $r$ is called acyclic if $D^{-} \neq \varnothing$ for all $D \in \mathcal{D}$. It is said to be realizable if there is a function $\varphi: X \rightarrow \mathbb{R}^{r}$ so that a signed set $D$ on $X$ with $|\bar{D}|=r$ is in $\mathcal{D}$ if and only if 0 is a positive linear combination of the points $\left\{\varphi(x): x \in D^{+}\right\} \cup\left\{-\varphi(x): x \in D^{-}\right\}$. In this case we say that the set $\varphi(X)$ realizes $(X, \mathcal{D})$.

For a collection $\mathcal{D}$ of signed subsets of $X$, and $x \in X$, define $\mathcal{D} / x$ to be the collection $\left\{\left(D^{+} \backslash\{x\}, D^{-} \backslash\{x\}\right): x \in \bar{D}\right\}$. If $\mathcal{O}=(X, \mathcal{D})$ is a uniform oriented matroid of rank $r$ and $x \in X$, then $\mathcal{O} / x=(X \backslash\{x\}, \mathcal{D} / x)$ is a uniform oriented matroid of rank $r-1$, called the oriented matroid obtained from $\mathcal{O}$ by contracting $x$.

Suppose now that $\mathcal{O}=(X, \mathcal{D})$ is an acyclic uniform oriented matroid. One can define a function $c l_{\mathcal{C}}: 2^{X} \rightarrow 2^{X}$ by $x \in \operatorname{cl}_{C}(A)$ if $x \in A$ or $\left(D^{+},\{x\}\right) \in \mathcal{D}$ for some subset $D^{+}$of $A$. Then [2] shows that $\mathcal{C}=\left\{C \subseteq X: c_{\mathcal{C}}(C)=C\right\}$ is a convex geometry on $X$. In this case we say that $\mathcal{C}$ is realized by $\mathcal{O}$.

Two different notions for "realizability" of a convex geometry $(X, \mathcal{C})$ have been presented. We show next how they are related. Suppose that $\mathcal{O}=(X, \mathcal{D})$ is an acyclic uniform oriented matroid of rank $r$, and that there is a function $\varphi: X \rightarrow \mathbb{R}^{r}$ so that $\varphi(X)$ realizes $\mathcal{O}$. Because $\mathcal{O}$ is acyclic, one can assume that the set $\varphi(X)$ is contained in an $(r-1)$-dimensional affine subspace of $\mathbb{R}^{r}$. If $(X, \mathcal{C})$ is a convex geometry realized by $\mathcal{O}$, then $\varphi(X)$ is also a realization of $(X, \mathcal{C})$. Thus "realization of $(X, \mathcal{C})$ by an acyclic rank $r$ oriented matroid" is implied by "realization of $(X, \mathcal{C})$ by a set of points of $R^{r-1}$." Unless specific reference is made to an oriented matroid that may not be realizable, realizability of a convex geometry can be assumed to be the stronger notion of realizability by a set of points in the plane.

If $\mathcal{O}=(X, \mathcal{D})$ is an acyclic uniform oriented matroid of rank 3 , and $x \in X$, then $\mathcal{O} / x$ is a uniform oriented matroid of rank 2. It is known (Corollary 8.3.3 of [1]) that every rank 2 oriented matroid is realizable. Thus, for every $x \in X$ we have a function $\bar{\varphi}_{x}: X \backslash\{x\} \rightarrow \mathbb{R}^{2}$ so that a signed set $D$ on $X \backslash\{x\}$ is in $\mathcal{D} / x$ if and only if 0 is a positive linear combination of the three points in $\left\{\bar{\varphi}_{x}(y): y \in D^{+}\right\} \cup\left\{-\bar{\varphi}_{x}(y): y \in D^{-}\right\}$. 
Proposition 4.3. IfC is a convex geometry on $X$, realized by an acyclic uniform oriented matroid $\mathcal{O}=(X, \mathcal{D})$ of rank 3 , then $\mathcal{C}$ is locally planar.

Proof. For each $x \in X$, let $\varphi_{x}: X \rightarrow \mathbb{R}^{2}$ satisfy $\varphi_{x}(y)=\bar{\varphi}_{x}(y)$ if $y \neq x$ and $\varphi_{x}(x)=0$. Then, for each $A \subseteq X, x \in c l_{A} \mathcal{C}$ if and only if $\varphi_{x}(x)$ is in the convex hull of $\left\{\varphi_{x}(y): x \in A\right\}$.

Problem 4.4. If $\mathcal{C}$ is a locally planar convex geometry on $X$, must there be an acyclic uniform oriented matroid $\mathcal{O}$ of rank 3 so that $\mathcal{C}$ is realized by $\mathcal{O}$ ?

For oriented matroids, it is known that if $\mathcal{D}$ is a collection of signed sets on $X$ satisfying (1)-(4) of Definition 4.2 with $r=3$, and each of the pairs $(X \backslash\{x\}, \mathcal{D} / x)$ for $x \in X$ is a uniform oriented matroid of rank 2 , then $(X, \mathcal{D})$ is a uniform oriented matroid of rank 3. See [17] or the discussion following Corollary 3.6.4 of [1].

Suppose $\mathcal{C}$ is a convex geometry on $X, \bar{D}$ is a four-element subset of $X$, and no element of $\bar{D}$ is in the closure of the three others. The definition of local planarity does not tell how to assign a signed set $D$ to $\bar{D}$. One would like to be able to define such signed sets $D$ so that all of the collections $\mathcal{D} / x$ define uniform oriented matroids of rank 2 , but it is not clear that this is possible.

\section{Examples Generated by Regions on a Disk}

Let $B=\left\{(x, y) \in \mathbb{R}^{2}: x^{2}+y^{2} \leq 1\right\}$, and let $E=\left(e_{1}, e_{2}, \ldots, e_{n}\right)$ be a sequence of points on the boundary of $B$ in clockwise order around $B$. Assume that no three of the segments connecting points in $E$ intersect in the interior of $B$. It is known (see [11] for three proofs) that the line segments connecting points of $E$ create $\left(\begin{array}{l}n \\ 4\end{array}\right)+\left(\begin{array}{l}n \\ 2\end{array}\right)+\left(\begin{array}{l}n \\ 0\end{array}\right)$ two-dimensional regions in $B$. Let $X$ be the set of regions.

Figure 5.1 shows such a set $X$ of regions for $n=5$. We will presently define a locally planar convex geometry $(X, \mathcal{C})$ generated by five linear orders so that the odd sets in the figure are the sets $\chi(x)$ as defined in Section 2. The five linear orders will, in fact, be those of Example 3.3.

For $x, y \in X$ we introduce the notation $\left.x\right|_{i} ^{j} y$ to mean that the line segment from $e_{i}$ to $e_{j}$ separates $x$ and $y$, and that $x$ is on the same side of this segment as the arc of the unit circle that goes clockwise from $e_{i}$ to $e_{j}$. We also write $\left.x\right|_{i} y$ if $\left.x\right|_{i} ^{j} y$ for some $j$, and say that $e_{i}$ separates $x$ and $y$ if $\left.x\right|_{i} y$ or $\left.y\right|_{i} x$. Finally, we say $\left.x\right|_{i} ^{j}$ if $x$ is on the same side of the line segment from $e_{i}$ to $e_{j}$ as the arc of the unit circle that goes clockwise from $e_{i}$ to $e_{j}$. Whenever we consider a sequence of the form $(i, i-1, \ldots)$ we assume that the elements are taken modulo $n$.

Definition 5.1. For $i \in\{1,2, \ldots, n\}$ and $x, y \in X$, define $x>y$ in $L_{i}$ if $\left.x\right|_{k} y$ where $k$ is the first element of the sequence $(i, i-1, \ldots)$ so that $e_{k}$ separates $x$ and $y$.

Proposition 5.2. For each $i \in\{1,2, \ldots, n\}, L_{i}$ is a linear order on $X$. 


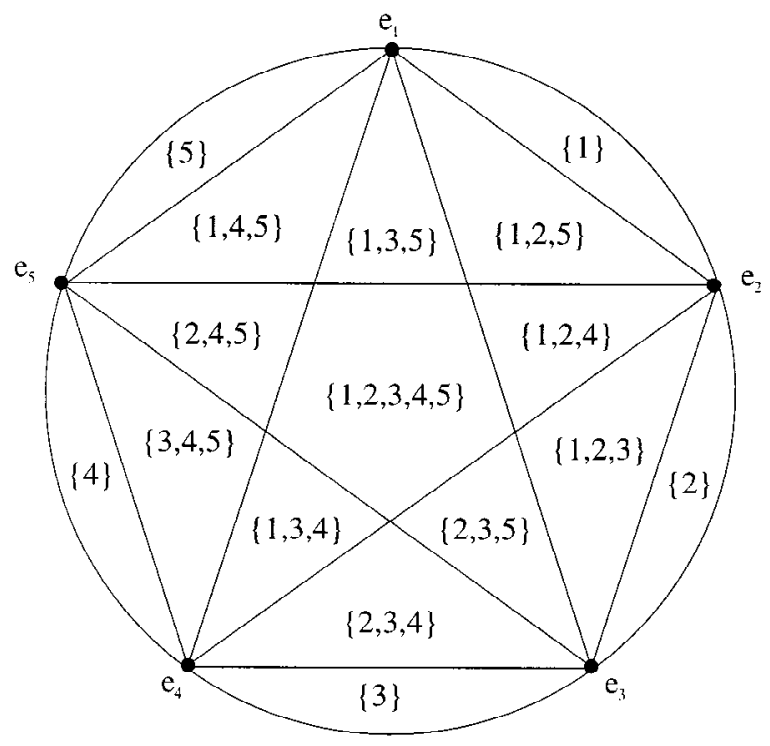

Fig. 5.1. The set of regions for $n=5$.

Indeed, we can write, for each $x \in X$, a vector $\left(x_{1}, x_{2}, \ldots, x_{n}\right)$, where $x_{j}=k-j$ (modulo $n$ ) if $\left.\right|_{j} ^{k} x$ and $\left.x\right|_{j} ^{k+1}$. Then $L_{i}$ is just lexicographic order, starting at the $i$ th component of $\left(x_{1}, x_{2}, \ldots, x_{n}\right)$ and going backwards.

Let $(X, \mathcal{C})$ be the convex geometry generated by $L_{1}, L_{2}, \ldots, L_{n}$. Note that each of the orders $L_{i}$ has a different maximal element. It follows that $(X, \mathcal{C})$ is not generated by fewer than $n$ linear orders of $X$.

Lemma 5.3. For $x, y \in X$, the $\operatorname{set}\left\{e_{i}: y>x\right.$ in $\left.L_{i}\right\}$ is an interval in the cyclic ordering of $E$.

Proof. Suppose first that $y>x$ on the orders $L_{i}$ and $L_{j}$, where $1 \leq i<j \leq n$, and that $e_{i}$ and $e_{j}$ separate $y$ and $x$. Then there exist $k, l$ so that $\left.y\right|_{i} ^{k} x$ and $\left.y\right|_{j} ^{l} x$. A quick sketch shows that $e_{k}$ and $e_{l}$ are either both in the arc from $e_{i}$ to $e_{j}$ or both in the arc from $e_{j}$ to $e_{i}$. Without loss of generality, assume that $i<k, l<j$. If $l<k$, then the segments $\overline{e_{i} e_{k}}$ and $\overline{e_{j} e_{l}}$ cross in the interior of the disk at a point $\alpha$. If $m<i$ or $m>j$ and $s \neq m$, then $\left.\right|_{m} ^{s} x$ whenever $\left.\right|_{m} ^{s} \alpha$ and $\left.y\right|_{m} ^{s}$ whenever $\left.\alpha\right|_{m} ^{s}$. In neither case can we have $\left.x\right|_{m} ^{s} y$. It follows that $y>x$ on $L_{m}$ whenever $m<i$ or $m>j$. Next, suppose that $y>x$ on the orders $L_{u}$ and $L_{v}$, that the first element of the sequence $e_{u}, e_{u-1}, \ldots$ that separates $y$ and $x$ is $e_{i}$, and that the first element of the sequence $e_{v}, e_{v-1}, \ldots$ that separates $y$ and $x$ is $e_{j}$. Then either $y>x$ on $L_{j}, L_{j+1}, \ldots, L_{i}$ or $y>x$ on $L_{i}, L_{i+1}, \ldots, L_{j}$. In both cases there is an interval in the cyclic order of $E$, from $e_{u}$ to $e_{v}$ or from $e_{v}$ to $e_{u}$, such that $y>x$ on $L_{t}$ for each $e_{t}$ in the interval.

Note that the above proof does not require that the line segments $\overline{e_{j} e_{l}}$ and $\overline{e_{i} e_{k}}$ be straight, only that they cross in exactly one point. 


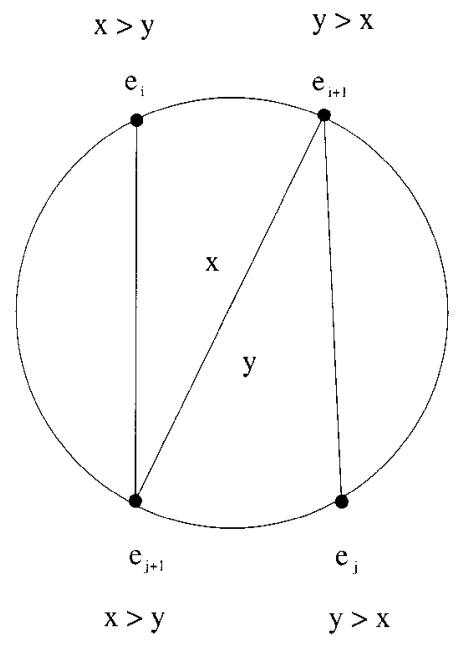

Figure 5.2. See Lemma 5.4.

Lemma 5.4. Suppose that $y>x$ on $L_{j}, x>y$ on $L_{j+1}, x>y$ on $L_{i}$, and $y>x$ on $L_{i+1}$. Then $\left.x\right|_{i+1} ^{j+1} y,\left.\right|_{j+1} ^{i} x$, and $\left.y\right|_{j} ^{i+1}$.

Proof. (See Fig. 5.2. Note that it is possible that $j+1=i$ and $i+1=j$.) From the definition of the orderings $L_{i+1}$ and $L_{j+1}$ it is clear that $\left.x\right|_{j+1} y$ and $\left.y\right|_{i+1} x$. Let $k$ be such that $\left.x\right|_{j+1} ^{k} y$. Then $k$ cannot be in the sequence $j+2, \ldots, i$ due to the previous lemma. Thus $\left.\right|_{j+1} ^{i+1} y$. A symmetric argument shows that $\left.\right|_{i+1} ^{j+1} x$, so $\left.x\right|_{i+1} ^{j+1} y$. Because $x>y$ on $L_{i}$ and $\left.y\right|_{i} ^{j+1}$, we must also have $\left.x\right|_{i} ^{j+1}$, which is the same as $\left.\right|_{j+1} ^{i} x$. By symmetry, $\left.y\right|_{j} ^{i+1}$.

Proposition 5.5. Let $x, y, z$ be distinct elements of $X$. The set $\left\{e_{i}: z>\{x, y\}\right.$ on $\left.L_{i}\right\}$ is a nonempty interval in the cyclic ordering of $E$.

Proof. Let $x, y$, and $z$ be distinct elements of $X$. We can assume that $x$ and $y$ are as in Lemma 5.4. Consider Fig. 5.2. If $\left.x\right|_{j+1} ^{i}$, then $z>x>y$ on $L_{j+1}$. Suppose $\left.\left.\right|_{j+1} ^{i+1} z\right|_{j} ^{i+1}$. If then $y>z$ on $L_{i+1}, L_{i+2}, \ldots, L_{j}$, it would follow from Lemma 5.4 that $\left.z\right|_{k} ^{l} y$ for some $k$ and $l$ so that the sequence $\left(e_{j+1}, e_{k}, e_{l}, e_{i+1}\right)$ is encountered as we go clockwise from $e_{j+1}$ to $e_{i+1}$ A quick look at Fig. 5.2 shows that this is not possible. Thus $z>y>x$ on $L_{k}$ for some $k$ in the sequence $(i+1, i+2, \ldots, j)$. For the case $\left.z\right|_{i+1} ^{j}$ and the case $\left.\left.\right|_{j+1} ^{i} z\right|_{j+1} ^{i+1}$ we similarly find orders on which $z>\{x, y\}$. This means that $z \notin c l_{\mathcal{C}}(\{x, y\}$.

Because $x, y$, and $z$ were arbitrary, we see that all two-element subsets of $X$ are in $\mathcal{C}$. Proposition 5.5 therefore shows that $\mathcal{C}$ is 2 -free.

We would now like to construct a collection $\mathcal{D}$ of signed sets on $X$ that we will later prove to be the set of circuits of a uniform oriented matroid of rank 3. Let $A=\{x, y, z, w\}$ be a subset of $X$. Because $X$ is 2-free, we either have one of the elements of $A$, say $x$, in 
the closure of the $A \backslash x$, or $A$ is an independent set. If $x$ is in the closure of $\{y, z, w\}$, then $\mathcal{D}$ will include the signed sets $(\{x\},\{y, z, w\})$ and $(\{y, z, w\},\{x\})$. If $A$ is independent, then each of $x, y, z$, and $w$ is the largest element of $A$ on at least one of the $L_{i}$. For each $t \in A$ let $L_{t}$ be an order on which $t$ is the largest element of $A$. Suppose the sequence $\left(e_{x}, e_{y}, e_{z}, e_{w}\right)$ is as in the clockwise order of $E$. In that case, $\mathcal{D}$ will include the signed sets $(\{x, z\},\{y, w\})$ and $(\{y, w\},\{x, z\})$. By Proposition 5.5, the set of $e_{i}$ for which a given member of $A$ is the largest element of $A$ on $L_{i}$ is an interval in the cyclic order of $E$. This implies that our signed sets are well defined. We now have a collection $\mathcal{D}$ that satisfies (1)-(4) of Definition 4.2 with $r=3$. We need to show that (5) is also satisfied. Note that (5) only involves sets of five elements of $X$ when $r=3$. We will therefore have proved that (5) holds if we can show that the restriction of $\mathcal{D}$ to any five-element subset of $X$ yields the set of circuits of an oriented matroid that is realizable in $\mathbb{R}^{3}$.

Suppose that $A=\{x, y, z, u, v\} \subseteq X$. We say that an element $t$ of $A$ is extreme for $A$ if $t$ is the largest element of $A$ on some order $L_{i}$. There will be three cases, depending on the number of extreme elements for $A$.

Suppose that for each $t \in A$ there is an order $L_{t}$ on which $t$ is the largest element of $A$. Suppose that $\left(e_{x}, e_{y}, e_{z}, e_{u}, e_{v}\right)$ is as in the clockwise order of $E$. Then $\mathcal{D}=\{(\{x, z\},\{y, u\}),(\{y, u\},\{x, z\}),(\{x, z\},\{y, v\}),(\{y, v\},\{x, z\}),(\{x, u\},\{y, v\})$, $(\{y, v\},\{x, u\}),(\{x, u\},\{z, v\}),(\{z, v\},\{x, u\}),(\{y, u\},\{z, v\}),(\{z, v\},\{y, u\})\}$. This is the set of circuits of an oriented matroid that is realizable by a set of points that are the vertices of a convex pentagon. (This pentagon would be contained in a plane in $\mathbb{R}^{3}$ that does not contain the origin.)

Suppose that for each $t \in A \backslash\{v\}$ there is an order $L_{t}$ on which $t$ is the largest element of $A \backslash\{v\}$, and that $v \in \operatorname{cl}_{\mathcal{C}}(A \backslash\{v\})$. We can assume that $e_{x}, e_{y}, e_{z}, e_{u}$ is as in the clockwise order of $E$. Note that if $y$ is largest in $A \backslash\{v\}$ on an order $L_{i}$, then $u$ may not be second largest, for in that case the collection of $e_{j}$ on which $u>\{x, z\}$ would not be an interval in the cyclic ordering of $E$. There must either be an order on which $u$ is the largest and $v$ is the second largest element of $A$, or an order on which $y$ is the largest and $v$ is the second largest element of $A$, but not both, for otherwise $\left\{e_{i}: v>\{x, z\}\right.$ on $L_{i}$ would not be a nonempty interval in the cyclic order of $E$. We can assume that there is an order in which $u$ is the largest and $v$ is the second largest element of $A$, and similarly that there is an order in which $z$ is the largest and $v$ is the second largest element of $A$. Thus the sets $\{x, y, z, v\}$ and $\{x, y, v, u\}$ are independent, and we have $v \in \operatorname{cl}_{\mathcal{C}}(\{y, z, u\}), v \in \operatorname{cl}_{\mathcal{C}}(\{x, z, u\})$. The circuits are $\mathcal{D}=\{(\{x, z\},\{y, u\}),(\{y, u\},\{x, z\}),(\{x, z\},\{y, v\}),(\{y, v\},\{x, z\}),(\{x, v\}\{y, u\})$, $(\{y, u\},\{x, v\}),(\{v\},\{y, z, u\}),(\{y, z, u\},\{v\}),(\{v\},\{x, z, u\}),(\{x, z, u\},\{v\})\}$. This is the set of circuits realized by the set of vertices of a convex quadrilateral and a point in its interior.

Finally, assume that $x, y, z$ are independent, and that $\{u, v\} \subseteq \operatorname{cl}_{\mathcal{C}}(\{x, y, z\})$.

Lemma 5.6. $v$ is in at most one of the sets $c l_{\mathcal{C}}(\{x, y, u\}), c l_{\mathcal{C}}(\{x, z, u\})$, and $c l_{\mathcal{C}}(\{y, z, u\})$.

Proof. Suppose that $v \in c l_{\mathcal{C}}(\{x, y, u\})$. There is an order $L_{k}$ on which $u>\{x, y\}$. We must then have $z>u>\{x, y, v\}$ on $L_{k}$. There must be an order $L_{i}$ on which $v>\{x, u\}$, and $y$ is the largest element of $A$ in $L_{i}$. There is also an order $L_{j}$ on which $u>\{x, z\}$, and $y$ is the largest element of $A$ in $L_{j}$. If either $v>z$ in $L_{i}$ or $v>u$ in $L_{j}$, then 
$v \notin c l_{\mathcal{C}}(\{x, z, u\})$. Assume that $y>z>v>\{x, u\}$ in $L_{i}$ and $y>u>v>\{x, z\}$ in $L_{j}$. Because $u \notin c l_{\mathcal{C}}(\{y, z\})$ and $v \notin c l_{\mathcal{C}}(\{y, u\})$, there must be an $L_{l}$ on which $x>u>\{y, z\}$ and an $L_{m}$ on which $x>v>\{y, z\}$. Now consider the order in which $e_{k}, e_{i}, e_{j}, e_{l}, e_{m}$ appear in the clockwise or counterclockwise ordering of $E$. Within this set, $e_{i}$ and $e_{j}$ are adjacent because $y$ is largest in each of them, and $e_{l}, e_{m}$ are similarly adjacent. If we meet the subsequence $\left(e_{l}, e_{i}, e_{j}, e_{k}\right)$, then the $\left\{e_{p}: z>u\right.$ in $\left.L_{p}\right\}$ is not an interval. The other possibility is that we encounter the sequence $\left(e_{m}, e_{j}, e_{i}, e_{k}\right)$ (here $e_{j}$ and $e_{i}$ are reversed). In that case, $\left\{e_{p}: u>v\right\}$ in $L_{p}$ is not an interval. We can therefore conclude that $v \notin c l_{\mathcal{C}}(\{x, z, u\})$. An analogous argument shows that $v \notin c l_{\mathcal{C}}(\{y, z, u\})$.

Because there is at most one $t \in\{x, y, z\}$ so that $v \in c l_{\mathcal{C}}\{x, y, z, u\} \backslash\{t\}$ and at most one $t \in\{x, y, z\}$ so that $u \in c l_{\mathcal{C}}\{x, y, z, v\} \backslash\{t\}$, there must be a $t \in\{x, y, z\}$ so that $\{x, y, z, u, v\} \backslash\{t\}$ is independent. Assume that $\{x, y, u, v\}$ is independent. For each $t \in\{x, y, u, v\}$, let $L_{t}$ be an order in which $t$ is the largest element of $\{x, y, u, v\}$. Note that $z>u$ in $L_{u}$ and $z>v$ in $L_{v}$, so when we encounter $\left\{e_{x}, e_{y}, e_{u}, e_{v}\right\}$ in the clockwise or counterclockwise order of $E$, then $e_{u}$ and $e_{v}$ will be adjacent. We can therefore assume that the circular ordering of these elements is $\left(e_{x}, e_{y}, e_{u}, e_{v}\right)$. If $v>u$ on an order $L_{i}$ in which $y$ is the largest of $\{x, y, u, v\}$, then $\left\{e_{j}: v>u\right\}$ in $L_{j}$ will not be an interval in the cyclic ordering of $E$. Thus $v \in c l_{\mathcal{C}}(\{x, u, z\})$. Similarly, $u \in c l_{\mathcal{C}}(\{y, v, z\})$. We thus have the circuits $\mathcal{D}=\{(\{u\},\{x, y, z\}),(\{x, y, z\},\{u\}),(\{v\},\{x, y, z\}),(\{x, y, z\},\{v\})$, $(\{x, u\},\{y, v\}), \quad(\{y, v\},\{x, u\}), \quad(\{v\},\{x, u, z\}), \quad(\{x, u, z\},\{v\}), \quad(\{u\},\{y, v, z\})$, $(\{y, v, z\},\{u\})\}$. This is the set of circuits one gets from a triangle with vertices $x, y, z$ and points $u$ and $v$ in its interior, when the points $x, y, u, v$ form a convex quadrilateral.

Proposition 5.7. The convex geometry $(X, \mathcal{C})$ given by the method of this section with $n=5$ is not realizable by a set of points in the plane.

Proof. Let the regions of the disk be labeled as in Fig. 5.1. Suppose that there is a function $\varphi: X \rightarrow \mathbb{R}^{2}$ so that $\varphi(X)$ realizes $(X, \mathcal{C})$. Note that $A=\{\{1,2,5\},\{1,2,3\},\{2,3,4\}$, $\{3,4,5\},\{1,4,5\}\}$ is independent, and so $\varphi(A)$ is the set of vertices of a convex pentagon. Similarly, $B=\{\{1,2,4\},\{2,3,5\},\{1,3,4\},\{2,4,5\},\{1,3,5\}\}$ is independent. Furthermore, each element of $B$ is in $\operatorname{cl}_{\mathcal{C}}(A)$, so $\varphi(B)$ is in the convex hull of $\varphi(A)$. Note that $\{1,3,5\} \in \operatorname{cl}_{\mathcal{C}}(\{\{1,2,5\},\{1,4,5\},\{3,4,5\}\}) \cap c_{\mathcal{C}}(\{\{1,2,4\},\{1,4,5\},\{2,3,5\}\})$. These considerations, together with the cyclic symmetry, imply that the image of $A \cup B$ must be as in Fig. 5.3. It now becomes impossible to place $\varphi(\{1,2,3,4,5\})$ in the figure, because $\{1,2,3,4,5\}$ is in $\operatorname{cl}_{\mathcal{C}}(\{\{1,2,3\},\{1,3,4\},\{2,4,5\}\}) \cap \operatorname{cl}_{\mathcal{C}}(\{\{2,3,4\},\{2,4,5\}$, $\{1,3,5\}\}) \cap \operatorname{cl}_{\mathcal{C}}(\{\{3,4,5\},\{1,3,5\},\{1,2,4\}\}) \cap \operatorname{cl}_{\mathcal{C}}(\{\{1,4,5\},\{1,2,4\},\{2,3,5\}\}) \cap$ $c l_{\mathcal{C}}(\{\{1,2,5\},\{2,3,5\},\{1,3,4\}\})$.

The nonrealizability of this example together with Proposition 3.4 yield the following.

Proposition 5.8. There is no convex geometry of convex dimension $n$ with $2^{n-1}$ points that is realizable by a nondegenerate set of points in the plane, for $n \geq 5$.

Figure 5.4 shows one half of an arrangement of pseudocircles that represents an 


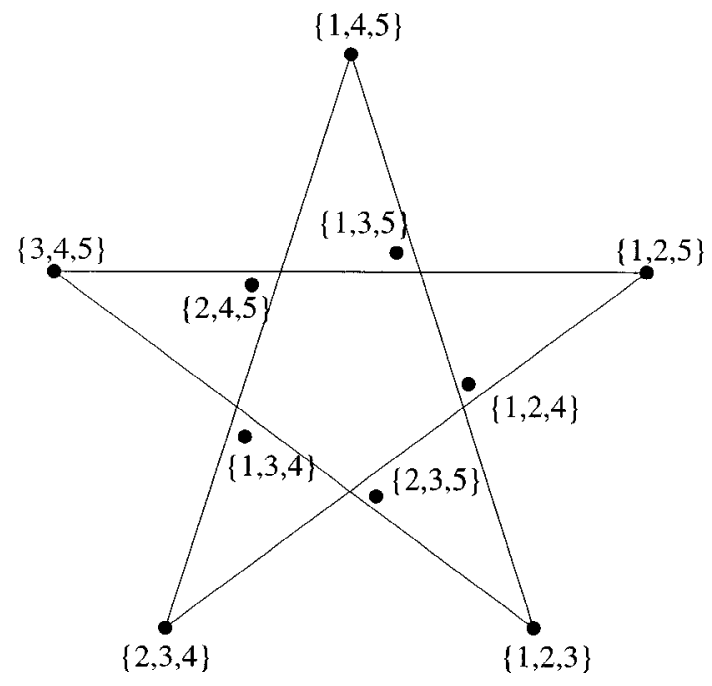

Fig. 5.3. See Proposition 5.7.

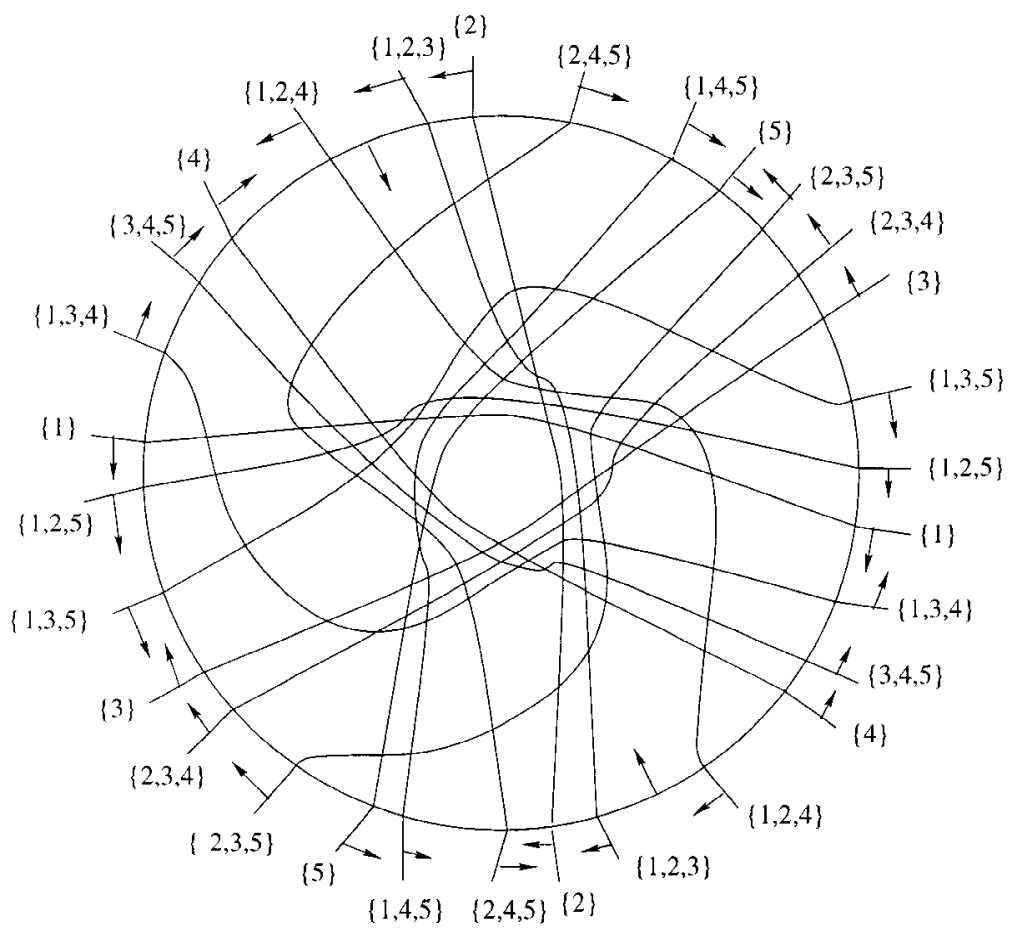

Fig. 5.4. Pseudocircle arrangement for $n=5$. 
oriented matroid realizing our $n=5$ example. One should think of the disk as the northern hemisphere of $S^{2}$, with the outside circle on the equator. On the southern hemisphere we have a copy of the drawing, with the arrows reversed. The curves represent elements of the convex geometry. The elements have been labeled by the odd sets $\chi(x)$ as in Fig. 5.1. The outside circle represents the center region of Fig. 5.1, labeled $\{1,2,3,4,5\}$. Given elements $x, y, z, w$ of $X$, one can read the associated circuit of $\mathcal{D}$ as follows: Delete all of the circles except those representing $x, y, z, w$. If all of the arrows point to a cell that has four sides, then $\{x, y, z, w\}$ is independent. If the circles are encountered in the order $(x, y, z, w)$ as one goes around the edge of this cell, then $\mathcal{D}$ contains the circuits $(\{x, z\},\{y, w\})$ and $(\{y, w\},\{x, z\})$. If the cell pointed to by all the arrows has only three sides, representing $x, y, z$, then $\mathcal{D}$ contains the circuits $(\{w\},\{x, y, z\})$ and $(\{x, y, z\},\{w\})$.

Because the convex dimension of this convex geometry is 5 , it follows that $X$ contains no independent set of size 6 . The geometric interpretation of this statement is that if one deletes ten of the pseudocircles of Fig. 5.4, then the region of $S^{2}$ that is pointed to by all of the arrows cannot be adjacent to all of the six remaining pseudocircles (see [10]).

\section{Acknowledgments}

We thank Serkan Hosten, Stefan Felsner, and the referees for constructive criticisms of an earlier version of this paper.

\section{References}

1. A. Björner, M. Las Vergnas, B. Sturmfels, N. White and G. M. Ziegler, Oriented Matroids, Cambridge University Press, Cambridge, 1993.

2. P. H. Edelman and R. E. Jamison, The theory of convex geometries, Geom. Dedicata 19 (1985), 247-270.

3. P. H. Edelman and D. G. Larman, On characterizing collections arising from $n$-gons in the plane, Geom. Dedicata 33 (1990), 83-89.

4. P. H. Edelman and M. E. Saks, Combinatorial representation and convex dimension of convex geometries, Order 5 (1988), 23-32.

5. P. Erdős and N. Hindman, Enumeration of intersecting families, Discrete Math. 48 (1984), 61-65.

6. P. Erdős and G. Szekeres, A combinatorial problem in geometry, Compositio Math. 2 (1935), 463-470.

7. P. Erdős and G. Szekeres, On some extremum problems in elementary geometry, Ann. Univ. Sci. Budapest. Eötvös, Sect. Math., 3 (1960/61), 53-62.

8. J. Folkman and J. Lawrence, Oriented Matroids, J. Combin. Theory Ser. B 25 (1978), 199-236.

9. J. E. Goodman and R. Pollack, A combinatorial perspective on some problems in geometry, Congr. Numer. 32 (1981), 383-394.

10. J. E. Goodman and R. Pollack, A theorem of ordered duality, Geom. Dedicata 12 (1982), 63-74.

11. R. Honsberger, Mathematical Gems, Mathematical Assocociation of America, Washington, DC, 1973.

12. S. Hoşten and W. D. Morris, Jr., The order dimension of the complete graph, Discrete Math. 201 (1999), 133-139.

13. R. E. Jamison, Copoints in antimatroids, Congr. Numer. 29 (1980), 535-544.

14. A. D. Korshunov, Families of subsets of a finite set and closed classes of Boolean functions, Bolyai Soc. Math. Studies 3 (1994), 375-396. 
15. B. Korte and L. Lovász, Shelling structures, convexity and a happy end, Graph Theory and Combinatorics, Academic Press, London, 1984, pp. 219-232.

16. L. Lovász, Combinatorial Problems and Exercises, North-Holland, Amsterdam, 1979.

17. I. Streinu, Clusters of stars, Proc. ACM Symp. Comput. Geom. (1997), pp. 439-441.

18. G. Tóth and P. Valtr, A note on the Erdős-Szekeres theorem, Discrete Comput. Geom. 19 (1998), 457-459.

Received June 7, 1999, and in revised form April 18, 2000. Online publication September 22, 2000. 